\title{
Doença mental, mulheres e transformação social: um perfil evolutivo institucional de 1931 a 2000
}

\author{
Fábio Leite Gastal ${ }^{*}$ \\ Sérgio Soares Olivé Leite** \\ Erika Cristine Treptow*** \\ Stela Scaglioni Marini ${ }^{* * *}$ \\ Maurício Vargas Noal ${ }^{\star \star *}$ \\ Mario Arthur Rockenbach Binz\# \\ Milene Tombini do Amaral\#
}

\section{INTRODUÇÃO}

O século $X X$ trouxe mudanças reveladoras à humanidade, tanto nas áreas científica $e$ tecnológica quanto nas áreas educacional e social. Um dos segmentos mais envolvidos por essas mudanças e transformações foi o gênero feminino. As transformações modernizantes,

O presente trabalho foi apresentado no VIII Congresso Brasileiro de Clínica Médica, que se realizou nos dias 4 a 7 de setembro de 2005, sob a forma de pôster.

* Médico psiquiatra. Doutor em Medicina, Universidad de la República Oriental del Uruguay (UROU), Montevidéu, Uruguai. Doutor em Psiquiatria, Universidade Federal de São Paulo - Escola Paulista de Medicina (UNIFESP-EPM), São Paulo, SP. Professor convidado, Programa de PósGraduação de Medicina Tropical, Universidade Federal de Minas Gerais (UFMG), Belo Horizonte, MG. Superintendente, Organização Nacional de Acreditação (ONA), Brasília, DF

${ }^{\star *}$ Médico psiquiatra. Professor titular de Psiquiatria, Universidade Católica de Pelotas (UCPel), Pelotas, RS

*** Médico(a), UCPel, Pelotas, RS. Ex-estagiário(a), Programa de Iniciação Científica, Conselho Nacional de Desenvolvimento Científico e Tecnológico (CNPq).

\# Acadêmico de Medicina, UCPel, Pelotas, RS. Estagiário, Programa de Iniciação Científica, CNPq

Grupo de Pesquisa CNPQ: "Avaliação de serviços, sistemas de informação e políticas de saúde", Escola de Medicina da UCPel e Clínica Olivé Leite, Pelotas, RS. após 1930, possibilitaram a configuração de uma nova feição da condição feminina. As mulheres tiveram o seu papel social redefinido à luz das mudanças ocorridas na família e nas condições sociais e econômicas. Conquistaram direitos políticos, asseguraram o acesso à educação e passaram a ganhar o espaço público do trabalho. O estabelecimento do novo padrão de atividade feminina permitiu a passagem da mulher das camadas médias do status anterior de esposa e de mãe para o status de trabalhadora. A busca de uma identidade própria e do reconhecimento social dessa identidade teve um impacto profundo sobre o modelo dominante de família baseado na ética do provedor. Conforme Singly: "O trabalho extra-doméstico, que era tido, até então, como uma atribuição masculina, passou a integrar o cotidiano feminino, fazendo com que elas passassem a assumir o desafio da conciliação casa-trabalho, o que trouxe conseqüências evidentes para as relações entre os gêneros. O celibato, a 'chefia feminina', as separações e os recasamentos transformaram-se em eventos cada vez mais freqüentes na vida das mulheres"1.

Recebido em 26/10/2005. Aceito em 12/09/2006. 
No Brasil, a taxa de atividade econômica feminina vem tornando-se cada vez maior. Estatísticas referentes à década de 70 mostram que a participação feminina na população economicamente ativa (PEA) aumentou de 18,5 para $26,9 \%$ no final da década de $80^{2,3}$. Essa tendência mantém-se até a atualidade, sendo que, em 2004 , o PEA feminino foi de $45,4 \%{ }^{3}$. O número de mulheres em profissões de alto prestígio (por exemplo, engenheiras, médicas, advogadas) cresceu aproximadamente $400 \%$, de 19.000 em 1970 para 95.800 em $1980^{2}$. Atualmente, em estados como o Rio Grande do Sul, $25,2 \%$ dos domicílios particulares permanentes estão sob responsabilidade de um representante do sexo feminino. Contudo, nos municípios de Santana do Livramento, Pelotas e Porto Alegre, os índices são ainda maiores, representando $30,3,31,4$ e $38,1 \%$, respectivamente, muito acima das médias brasileira $(24,9 \%)$ e gaúcha ${ }^{4}$.

A economia de Pelotas tem como característica a especialização de suas atividades, assentada em ramos tradicionais, como a agroindústria e a produção de alimentos. Esse último setor apresenta-se historicamente como espaço produtivo concentrador da força de trabalho feminina, devendo ser destacado o fato de que a produção de alimentos se relaciona, principalmente, à industrialização de conservas, atividade essa caracterizada pela sazonalidade. Isso significa que mudanças nesse setor afetam diretamente o perfil e a composição da força de trabalho feminina na indústria, com implicações sobre o conjunto dos empregos em Pelotas ${ }^{5}$.

Embora o movimento das mulheres esteja fazendo progressos, não se podem ocultar as conseqüências oriundas desse processo de mudanças. A tão desejada "liberdade feminina" traz consigo uma maior exposição ao risco de usar bebidas alcoólicas e drogas em geral, em especial as anfetaminas. Observa-se um crescente aumento do abuso de álcool entre as mulheres, sendo a prevalência em torno de $1,3 \%$. Todavia, essa proporção pode ser ainda maior, visto que a identificação do alcoolismo feminino em serviços de atenção primária se mostra deficiente e pouco valorizada ${ }^{6-8}$.

Ao contrário do que ocorre com os homens, a inserção feminina no mercado de trabalho é limitada por suas tarefas domésticas e familiares ${ }^{9}$. Além disso, as desigualdades em relação aos salários e ao tipo de atividade exercida ainda estão presentes na atualidade, levando muitas mulheres a se inserir no setor informal da economia. Um outro problema enfrentado pelas mulheres é o aumento do número de desempregadas, conforme dados apurados pela Pesquisa de Emprego e Desemprego da Região Metropolitana de Porto Alegre ${ }^{3}$.

$O$ presente estudo visa descrever o perfil da clientela feminina admitida em um serviço de internação psiquiátrica de 1931 a 2000, relacionando os dados encontrados com todo o processo formador da identidade feminina nos últimos anos.

\section{MATERIAL E MÉTODOS}

O presente trabalho baseia-se no levantamento de informações do banco de dados de um hospital psiquiátrico da cidade de Pelotas (RS). O modelo de pesquisa utilizado foi um estudo descritivo de uma série histórica de dados das pacientes do gênero feminino internadas desde a fundação do hospital, em $1^{\circ}$ de maio de 1931, até 31 de dezembro de 2000.

As informações clínicas e institucionais utilizadas estão organizadas num banco de dados estruturado em um sistema de registro de casos. Tais informações têm origem nos prontuários clínicos organizados no Serviço de Arquivo Médico e Estatística do hospital. O banco de dados informatizado foi criado em 1985 e contém as seguintes informações: nome, sexo, idade, nacionalidade, procedência, raça, estado civil, profissão/ocupação, data de internação, data de alta, diagnóstico, condições de alta, tratamento (referência geral) e condições da internação (custeio). Essas informações são atualizadas a cada internação, provenientes do Prontuário Eletrônico do Paciente (PEP/DADOS$\mathrm{H} / \mathrm{COL}$ ), disponibilizado na rede institucional e organizado de tal maneira que cada cliente internado no hospital seja registrado no banco como indivíduo-caso. Cada vez que ele reaparece no banco de dados (reinternação), constitui-se novo evento, mas não um novo registro ou caso. A utilização dessa sistemática permite a localização da pessoa com mais facilidade e auxilia o sistema no manejo dos dados, quando o mesmo indivíduo tem múltiplas internações.

A fim de analisar primeiramente os "casos", optou-se por trabalhar apenas com as primeiras internações de todas as pacientes do gênero feminino internadas no hospital no período de $1^{\circ}$ de maio de 1931 a 31 de dezembro de 2000 , totalizando uma população de 9.629 indivíduos/ casos. As variáveis incluídas no estudo foram as seguintes: cor, idade, estado civil, classe social, grupos nosológicos e ocupação. Para 
uma melhor análise evolutiva do perfil epidemiológico da população estudada, o período foi dividido por décadas, e cada uma delas foi analisada de forma individualizada para cada variável estudada. É válido lembrar que o hospital foi fundado em $1^{\circ}$ de maio de 1931; portanto, na primeira década estudada (1931 a 1940), não existem dados dos meses de janeiro, fevereiro, março e abril de 1931.

Os grupos nosológicos são baseados na Classificação Internacional de Doenças, em sua 9a edição, da Organização Mundial de Saúde (CID-9/OMS), seguem o mesmo perfil de agrupamento que foi proposto por Gastal ${ }^{10}$, convertidos e estruturados aos grupos nosológicos atuais da CID-10 (OMS) e descritos em tabelas organizadas por décadas. O uso de ambas as versões da CID ocorre porque a base de dados foi criada ainda na vigência da $9^{\text {a }}$ edição, sendo que todos os diagnósticos de versões mais antigas das classificações nosológicas e da $9^{a}$ para a $10^{\underline{a}}$ foram convertidos seguindo os algoritmos de conversão propostos pela OMS. Em caso de dúvidas, ocorria um processo de revisão das histórias clínicas, realizado por dois especialistas, para a geração de um diagnóstico conforme a citada classificação. A base de dados conta com os diagnósticos segundo as duas sistemáticas.

As variáveis sociodemográficas apresentam ocupação, setor profissional e classe social. Essas variáveis foram organizadas utilizando os critérios de Bronfman, modificados por Lombardi et al. ${ }^{11}$ e também utilizados por Victória et al. ${ }^{12}$.

As informações e dados das variáveis estudadas foram coletados da base de dados e organizados em tabelas, seguindo um modelo de análise estatística descritiva de dados, utilizando, para tal, software de tipo planilha eletrônica.

\section{RESULTADOS}

O perfil etário das pacientes internadas de maio de 1931 a dezembro de 2000 apresenta um predomínio da faixa etária de 26 a 45 anos (47,9\%), sendo as idades entre 26 e 35 anos responsáveis por $25,36 \%$ das internações. Não há registros de pacientes internadas com idade superior a 66 anos durante a década de 1931-1940. Com o passar dos anos, verifica-se progressiva ampliação da distribuição etária, de forma que, em 1991-2000, as pacientes com idade superior a 66 anos perfizeram $6,78 \%$ da população feminina internada (tabela 1).
Analisando o perfil racial das pacientes, constata-se a predominância de brancas $(91,3 \%)$ em relação às não-brancas. Essa situação manteve-se durante todas as décadas do estudo, apresentando variações entre 88,45 e $99,16 \%$. Todavia, observa-se um aumento progressivo da proporção de não-brancas, passando de $0,55 \%$ na década quarenta, para $11,55 \%$ na década de 90 .

Quanto ao estado civil, no decorrer das décadas, verifica-se maior presença de mulheres casadas $(44,9 \%)$ em relação às solteiras $(35,31 \%)$. Todavia, entre os anos de 1991-2000, essa proporção se inverteu: as solteiras representaram $54,45 \%$ das pacientes, e as casadas, $39,78 \%$. Além disso, observa-se aumento gradativo de internações de mulheres separadas. Nas quatro primeiras décadas, elas representavam não mais que $0,27 \%$ das pacientes, tendo atingido 3,36 e $8,83 \%$ nas duas últimas décadas, respectivamente. Do total de 9.629 de mulheres internadas, apenas oito $(0,08 \%)$ não apresentavam as informações relativas ao estado civil.

No que se refere à distribuição quanto aos setores profissionais, observa-se clara predominância de pacientes fora da população economicamente ativa (setor 13) no decorrer dos anos, especialmente de 1930 a 1960, quando perfizeram cerca de $96,71 \%$ das internações. Já a partir de 1960, verifica-se crescimento do percentual de mulheres empregadas no setor industrial, tendo 0 percentual passado de $0,98 \%$ na década de 60 para $20,12 \%$ na de 70 ; no entanto, observa-se novo declínio nas décadas subseqüentes, quando ocorreu o desaquecimento do setor industrial da cidade de Pelotas, principalmente em decorrência do fechamento de indústrias de alimentos e conservas.

Analisando a variável classe social, observa-se que o subproletariado representa $86,8 \%$ das internações. É evidente o crescimento do proletariado (classe 2) desde 1950, tendo atingido sua maior concentração entre os anos de 1961 e 1990, perfazendo $18,58 \%$ das internações na década de 19711980 e apresentando declínio entre 1991-2000 $(7,91 \%)$. Nas primeiras quatro décadas, não havia representantes da tecnocracia e burguesia empresariais entre as pacientes em primeira internação. Nas últimas décadas, porém, essas classes representaram 0,30 e $0,56 \%$ das internações, respectivamente. As perdas em relação à variável classe social foram de $0,12 \%$.

Em relação aos grupos nosológicos das pacientes, observa-se um maior número de 
internações por transtornos afetivos (28,6\%) e psicoses esquizofrênicas (25,2\%). As psicoses alcoólicas, as quais representavam menos de $1,5 \%$ das internações nas primeiras cinco décadas, mostraram aumento nas últimas, totalizando 6,38\% entre os anos de 1991 e 2000. Em contrapartida, a neurossífilis e o retardo mental apresentaram declínio significativo, com valores inferiores a $1 \%$ nas últimas três décadas. Analisando-se os transtornos neuróticos, observa-se que houve um aumento até a quarta década (10,33\%), com posterior queda, representando menos de $1 \%$ das internações na última década.

\section{DISCUSSÃO}

Os motivos para a admissão de mulheres em serviços psiquiátricos apresentaram uma série de mudanças no decorrer dos anos.

As variações observadas foram ditadas pela modificação do papel da mulher na sociedade. Desde a década de 60 , elas vêm ocupando postos de trabalho e passaram a exercer importante papel na economia ${ }^{13}$. A incorporação da mulher resultou de uma série de fatores, como aumento dos níveis de escolaridade, redução das taxas de fecundidade e expansão dos empregos. Essa

Tabela 1 - Perfil demográfico das pacientes internadas de maio de 1931 a dezembro de 2000

\begin{tabular}{lccccccc}
\hline Décadas & $31-40$ & $41-50$ & $51-60$ & $61-70$ & $71-80$ & $81-90$ & $91-00$ \\
\hline Idade (\%) & & & & & & & \\
< 16 & 0,77 & 1,1 & 2,27 & 2,78 & 2,98 & 2,52 & 1,53 \\
$16-25$ & 24,03 & 27,7 & 25,61 & 23,36 & 26,87 & 21,66 & 15,21 \\
$26-35$ & 40,31 & 25,76 & 26,65 & 28,75 & 24,48 & 23,82 & 25,48 \\
$36-45$ & 17,82 & 21,05 & 20,04 & 18,77 & 21,81 & 19,4 & 25,22 \\
$46-55$ & 10,07 & 14,95 & 13,42 & 13,47 & 14,47 & 15,3 & 15,93 \\
56-65 & 4,05 & 6,37 & 7,64 & 7,63 & 6,67 & 11,03 & 9,8 \\
66-75 & 0 & 1,66 & 2,68 & 3,32 & 1,93 & 4,71 & 5,05 \\
$>$ 75 & 0 & 1,38 & 1,23 & 0,8 & 0,7 & 1,42 & 1,73 \\
NC & 2,32 & 0 & 0,41 & 1,07 & 0,07 & 0,1 & 0 \\
Cor (\%) & & & & & & & \\
Brancas & 97,67 & 99,16 & 96,28 & 92,87 & 98,27 & 91,63 & 88,45 \\
Não-brancas & 2,52 & 0,55 & 0 & 0 & 0 & 0 & 11,54 \\
NC & 0,27 & 0 & 0 & 0 & 0 & 0,03 & 0 \\
Estado civil (\%) & & & & & & & \\
Solteiras & 27,9 & 30,74 & 33,67 & 28,12 & 36,91 & 32,84 & 42,18 \\
Casadas & 58,13 & 62,6 & 58,05 & 63,25 & 54,68 & 54,45 & 39,78 \\
Divorciadas & 0 & 0,27 & 0 & 0,26 & 1,29 & 3,36 & 8,83 \\
Viúvas & 10,85 & 6,37 & 8,05 & 8,26 & 7,09 & 9,24 & 8,98 \\
NC & 5,1 & 0 & 0,2 & 0,08 & 0 & 0,07 & 0,2 \\
Classe social (\%) & & & & & & 8,26 & \\
Subproletariado & 96,12 & 98,61 & 97,52 & 86,25 & 80,36 & 87,97 & 89,73 \\
Proletariado & 1,55 & 0,55 & 2,06 & 12,75 & 18,58 & 11,18 & 7,91 \\
Pequena burguesia & 1,55 & 0,83 & 0,2 & 0,8 & 0,91 & 0,76 & 1,17 \\
Tecnocracia & 0 & 0 & 0 & 0 & 0,07 & 0 & 0,3 \\
Burguesia empresarial & 0 & 0 & 0 & 0 & 0,03 & 0,03 & 0,56 \\
NC & 0,77 & 0 & 0,26 & 0,17 & 0,03 & 0,03 & 0,3 \\
\hline
\end{tabular}

* Subproletariado: assalariados, autônomos, ambulantes, construção civil, empregados domésticos, trabalhadores agrícolas, autônomos sem serviço ativo; proletariado: ocupações assalariadas nos setores profissionais: atividades industriais, comércio de mercadorias, serviços, serviços auxiliares de atividades econômicas, transportes e comunicações, social, administração pública, outras atividades e indústria de produtos alimentícios; pequena burguesia: comerciantes independentes, proprietários de negócios ativos, renda menor que nove salários mínimos ou até quatro empregados; tecnocracia (nova pequena burguesia): assalariados técnicos, profissionais, diretores, executivos de empresas; burguesia empresarial: proprietários, empregadores, renda igual ou maior que nove salários mínimos ou cinco ou mais empregados; NC = não consta. 
Doença mental, mulheres e transformação social - Gastal et al.

Tabela 2 - Perfil ocupacional e de setores profissionais das pacientes internadas de maio de 1931 a dezembro de 2000

\begin{tabular}{|c|c|c|c|c|c|c|c|}
\hline Décadas & $31-40$ & $41-50$ & $51-60$ & $61-70$ & $71-80$ & $81-90$ & $91-00$ \\
\hline \multicolumn{8}{|l|}{ Ocupação (\%) } \\
\hline OCUP1 & 1,55 & 0,83 & 0,2 & 0,8 & 1,58 & 3,65 & 5,56 \\
\hline OCUP2 & 0 & 0 & 0 & 0 & 0,03 & 0 & 0,05 \\
\hline OCUP3 & 0 & 0 & 0 & 0 & 0,1 & 0,03 & 0,76 \\
\hline OCUP4 & 0 & 0 & 0 & 0,98 & 1,22 & 1,35 & 2,29 \\
\hline OCUP5 & 0 & 0 & 0,41 & 0,8 & 0,7 & 0,65 & 0,56 \\
\hline OCUP6 & 0 & 0 & 0,2 & 1,61 & 2,66 & 2,15 & 1,73 \\
\hline OCUP7 & 1,55 & 0,27 & 1,23 & 7,72 & 26,27 & 15,49 & 5,97 \\
\hline OCUP8 & 96,12 & 98,61 & 96,48 & 85,08 & 65,57 & 74,38 & 80,54 \\
\hline NC & 0,77 & 0,27 & 1,44 & 2,96 & 1,82 & 2,26 & 2,5 \\
\hline \multicolumn{8}{|c|}{ Setores profissionais (\%) } \\
\hline SET1 & 0 & 0 & 0,2 & 0,08 & 0,84 & 2,95 & 4,9 \\
\hline SET2 & 0 & 0 & 0 & 0,44 & 0 & 0,03 & 0 \\
\hline SET3 & 0 & 0 & 0,2 & 0,44 & 2,45 & 3,21 & 1,17 \\
\hline SET4 & 0 & 0 & 0,82 & 0,98 & 20,12 & 9,86 & 1,17 \\
\hline SET5 & 2,32 & 0,83 & 0,2 & 3,5 & 3,61 & 2,26 & 2,5 \\
\hline SET6 & 0,77 & 0,27 & 0 & 1,25 & 2,77 & 2,08 & 2,8 \\
\hline SET7 & 0 & 0 & 0 & 0,44 & 1,12 & 1,2 & 2,09 \\
\hline SET8 & 0 & 0 & 0,2 & 0,17 & 0,17 & 0,03 & 0,3 \\
\hline SET9 & 0 & 0,27 & 1,65 & 3,95 & 2,88 & 3,47 & 3,42 \\
\hline SET10 & 0 & 0 & 0 & 0,53 & 0,28 & 0,29 & 0,56 \\
\hline SET11 & 0,77 & 0,27 & 0 & 0 & 0 & 0,14 & 0 \\
\hline SET12 & 0 & 0 & 0 & 2,87 & 0,03 & 0 & 0 \\
\hline SET13 & 95,34 & 98,33 & 96,48 & 85,08 & 65,64 & 74,38 & 80,54 \\
\hline NC & 0,77 & 0 & 0,2 & 0,17 & 0,03 & 0,03 & 0,2 \\
\hline
\end{tabular}

OCUP1 = proprietários; OCUP2 = administradores, gerentes; OCUP3 = profissionais de nível superior; OCUP4 = funções de escritório: corretores, contadores, secretários, datilógrafos, auxiliares de escritório, caixas; OCUP5 = trabalhadores manuais especializados (técnicos): aqueles que possuem curso técnico de nível médio; OCUP6 = trabalhadores manuais semi-especializados: mecânicos, eletricistas, armadores, soldadores, choferes, técnicos de indústria de transformação e construção civil, cabeleireiros; OCUP7 = trabalhadores manuais não-qualificados: pedreiros, lixeiros, serventes, empregados domésticos, estivadores, pescadores, operadores de máquinas, vendedores ambulantes, policiais (soldados), frentistas, office-boys, vigias, guardas-noturnos; OCUP8 = donas-de-casa, estudantes, pensionistas, aposentados, desempregados, doentes; NC = não consta; SET1 = agrícola: agropecuária, extração vegetal, pesca; SET2 = indústria de transformação: metalurgia, mecânica, moveleira, papel e celulose, curtumes, vestuário (mesmo domiciliares), química, plástico e petroquímica, têxtil, editorial, gráfica, farmacêutica e de produtos veterinários; SET3 = indústria de construção civil; SET4 = outras atividades industriais: extração mineral, serviços industriais de utilidade pública (energia elétrica, abastecimento de água, esgotos, limpeza pública e remoção de lixo); SET5 = comércio de mercadorias: supermercados, armazéns, feiras, magazines, atacados, açougues, postos de abastecimento, comércio de material usado, ferro-velho, vidros e papéis usados; SET6 = serviços: alojamento e alimentação (hotéis, pensões, restaurantes, bares, etc.), reparação e conservação (oficinas mecânicas), serviços pessoais e domiciliares (cabeleireiros, alfaiates domésticos), diversões, higiene e conservação de prédios, estiva; SET7 = serviçoS auxiliares de atividades econômicas: bancos, instituições financeiras, seguros, imobiliárias, cartórios, contábeis, jurídicos; SET8 = transportes e comunicações: transporte de passageiros, de carga, correios, telecomunicações, imprensa; SET9 = social: atividades comunitárias e sociais (seguro social, sindicatos, assistência social), serviços médicos e odontológicos (públicos e privados), ensino, hospitais, igrejas; SET10 = administração pública: serviços administrativos federais, estaduais, municipais, empresas públicas, legislativo, justiça, serviços de segurança pública (exército, marinha, aeronáutica, polícia e bombeiros); SET11 = outras atividades; SET12 = indústria de produtos alimentícios: alimentos, bebidas, frigoríficos, moinhos, padarias; SET13 = fora da população economicamente ativa: donas-de-casa, pensionistas, aposentados, desempregados. 
incorporação representou a trajetória de mulheres de distintos setores sociais - de um lado, as mulheres de baixa renda, que tiveram que se inserir no mercado de trabalho para garantir sobrevivência do grupo familiar e, de outro, as mulheres das camadas média e alta, que ingressaram na atividade econômica com vistas à busca de realização profissional ou visando à garantia de um nível de consumo ameaçado pelas mudanças econômicas em curso no país ${ }^{1}$. No entanto, a inserção feminina no processo produtivo uniu-se a outro importante pólo de identidade feminina, ligado à maternidade, à família e ao cuidado da casa, resultando em aumento da carga social a elas imposta. Atualmente, preconiza-se que uma mulher deve estar plenamente preparada para assumir as tarefas domésticas e realizar-se na maternagem dos filhos ${ }^{14}$. A dupla jornada motiva contradições e conflitos, estando estes provavelmente envolvidos na psicogênese e no desencadeamento dos transtornos psiquiátricos. Além disso, as pressões econômicas e sociais têm levado, cada vez mais, um maior número de mulheres a ingressarem no mercado informal de trabalho, o que representa uma oportunidade limitada e não reconhecida do uso de suas capacidades ${ }^{15}$. A incerteza sobre a sua situação de trabalho, como conseqüência da informalidade, da percepção de injustiça e discriminação e das menores oportunidades em relação às dos homens de uma inserção formal, pode estar negativamente associada ao bem-estar psicológico feminino ${ }^{16}$. Um outro problema enfrentado pelas mulheres refere-se às taxas crescentes de desemprego. A força de trabalho feminina tem sofrido mais com as adversidades que assolaram o mercado de trabalho nas últimas décadas, constituindo a maioria dos desempregados da região metropolitana de Porto Alegre. Esses resultados mostram que as mulheres ainda continuam enfrentando obstáculos para sua inserção laboral, pois, adicionalmente à falta de postos de trabalho originada na conjuntura econômica, ainda vigora, em suas relações com o mundo do trabalho, uma cultura discriminatória, oriunda de uma construção sociocultural tradicional de papéis atribuídos a homens e mulheres ${ }^{3}$. Está estabelecido, nas sociedades industrializadas, que os desempregados e suas famílias têm uma pior condição de saúde mental quando comparados com os que estão trabalhando ${ }^{16}$.

Tabela 3 - Grupos nosológicos das pacientes internadas de maio de 1931 a dezembro de 2000

\begin{tabular}{lccccccc}
\hline Décadas & $31-40$ & $41-50$ & $51-60$ & $61-70$ & $71-80$ & $\mathbf{8 1 - 9 0}$ & $91-00$ \\
\hline Grupos nosológicos (\%) & & & & & & & \\
SENIL & 3,1 & 6,92 & 5,78 & 6,01 & 2,07 & 3,65 & 5,05 \\
POT & 10,85 & 8,03 & 3,3 & 0,62 & 1,33 & 1,05 & 1,22 \\
POC & 0 & 0 & 0 & 0,26 & 3,09 & 3,72 & 5,66 \\
NSIF & 1,55 & 2,21 & 0,61 & 0,08 & 0 & 0 & 0 \\
RET & 4,65 & 3,04 & 6,4 & 1,61 & 0 & 0 & 0,3 \\
ALC-DEP & 0 & 0 & 0 & 0 & 0,17 & 0,1 & 0,81 \\
ALC-PSIC & 0 & 0,83 & 0 & 0,26 & 1,12 & 2,3 & 6,38 \\
TOX & 2,32 & 0 & 0,2 & 0 & 0,1 & 0,87 & 2,19 \\
ESQ & 24,8 & 32,13 & 31,4 & 26,5 & 26,8 & 24,58 & 20,58 \\
TAF & 25,58 & 22,16 & 26,65 & 28,3 & 24,76 & 32,44 & 31,05 \\
PSIC & 3,1 & 3,87 & 0,82 & 3,23 & 33,86 & 29,74 & 20,78 \\
NEUR & 3,87 & 7,2 & 8,67 & 10,33 & 0,42 & 0,03 & 0,35 \\
HIST & 4,65 & 3,32 & 7,02 & 10,96 & 4,95 & 0,36 & 0,61 \\
OUT & 8,52 & 9,14 & 7,02 & 8,35 & 1,19 & 1,05 & 4,85 \\
SDIAG & 6,97 & 1,1 & 2,06 & 3,41 & 0,1 & 0,03 & 0,1 \\
\hline
\end{tabular}

ALC-DEP = síndrome de dependência alcoólica; ALC-PSIC = psicoses alcoólicas; ESQ = psicoses esquizofrênicas; HIST = transtornos dissociativos (ou conversivos) - neurose histérica; NEUR = transtornos neuróticos; NSIF = neurossífilis; OUT = outros diagnósticos; POC = psicoses orgânicas crônicas; $P O T$ = psicoses orgânicas transitórias; $P S I C=$ psicose não-orgânica; $R E T$ = retardo mental; SDIAG = registros sem diagnóstico; SENIL = psicoses senis e pré-senis; TAF = transtornos afetivos; TOX = psicose e dependência causadas por drogas. 
O predomínio de pacientes internadas em idade produtiva (26-35 anos) reforça a existência de forte associação entre os fatores biológicos predisponentes e os fatores psicológicos relacionados à sobrecarga de trabalho e à maternidade com a presença de distúrbios psiquiátricos. Além disso, problemas de ordem profissional, familiar e socioeconômica preferencialmente culminam nessa faixa etária. Também é importante considerar o aumento de internações de pacientes com idade mais avançada. Esse fato deve-se, possivelmente, ao aumento da expectativa de vida, em especial na Região Sul do país. No Rio Grande do Sul, a expectativa de vida passou de 66,7 anos, em 1972, para 71,6 anos, em $2000^{4}$.

Com relação à cor, observa-se nítido predomínio da população branca sobre a nãobranca, fato explicado pela característica demográfica da região onde o estudo foi realizado. Todavia, na última década, foi demonstrado um aumento do número de pacientes não-brancas internadas. Isso se deve, talvez, ao crescente acesso da população em geral aos meios e serviços de saúde, graças ao surgimento dos sistemas de saúde e previdência social.

A maior proporção de mulheres casadas durante as primeiras décadas do estudo caracteriza a dependência econômica e os valores culturais da época. Todavia, após o advento dos movimentos femininos e a instituição do divórcio no Brasil (década de 70), essa relação modificou-se. Tornou-se freqüente a presença, na sociedade, de uma mulher independente e dinâmica, ao invés da figura submissa que ela representava no início do século passado. Conforme o Censo 2000, $31,5 \%$ dos lares de Pelotas estão sob a responsabilidade de mulheres, sendo que $26,5 \%$ destas são mães solteiras, viúvas ou separadas ${ }^{17}$.

Quanto aos setores profissionais, observase aumento significativo de mulheres inseridas no mercado de trabalho, especialmente no setor industrial, durante e após a década de 60, fato este que pode estar relacionado ao desenvolvimento da indústria de alimentos e conservas local nas décadas de 60 e $70^{13}$. O trabalho vem ganhando cada vez mais importância para as mulheres, concorrendo com o casamento e a maternidade como possibilidade de realização e ascensão social ${ }^{14}$. No entanto, observa-se um declínio no percentual de mulheres empregadas nas últimas décadas analisadas, fato justificado pela crise econômica e social ocorrida nos anos 80 , com forte impacto na região. A reestruturação atingiu fortemente os postos de trabalho em todos os setores da economia de Pelotas, mas é sobre a indústria que repercute de forma mais incisiva, com redução do contingente sazonal da força de trabalho. Se a redução do emprego sazonal, de menor estabilidade, que, em geral, ocupava as mulheres, e o aumento do emprego feminino nos demais setores que apresentam maior estabilidade demonstram, por um lado, ganhos quanto à qualidade dos postos de trabalho ofertados no conjunto da economia, por outro, mostram que os benefícios são carreados para as mulheres com mais alta escolarização e mais bem situadas no mercado, o que significa a penalização das mulheres com menor escolaridade, em geral advindas dos segmentos mais empobrecidos da população e que se submetem às rudes condições do trabalho sazonal ${ }^{5}$.

O subproletariado mostrou-se como classe social prevalente nas internações femininas. A valorização desigual do trabalho feminino em relação ao masculino pode ser responsável por essa prevalência. Dados da Comissão Econômica da América Latina e Caribe, referentes ao ano de 1996, mostraram que o salário médio feminino correspondia a apenas $68 \%$ da remuneração masculina ${ }^{18}$. Segundo levantamento do IBGE, em 2000, a referência nacional é de $67 \%$ no Brasil, sendo que, no Rio Grande do Sul, a disparidade apresenta-se um pouco menor: as mulheres recebem, em média, $29 \%$ a menos que os representantes do gênero masculino ${ }^{17}$. A incerteza apresentada pela mulher sobre sua situação de trabalho, devido à informalidade e à percepção de injustiça e discriminação, pode estar negativamente associada ao bem-estar psicológico feminino ${ }^{16}$.

O predomínio de internações por transtornos afetivos possivelmente relacionase à dupla jornada, responsável por acentuado sofrimento, sendo esta o estressor psicossocial relacionado à gênese de sintomas emocionais e/ou comportamentais significativos que caracterizam esse grupo nosológico. Entre as mulheres, associações entre os transtornos mentais comuns e emprego, estado civil e paridade são altamente complexas e variam de acordo com a transformação específica dos papéis sociais ocupados. Essa complexidade é claramente demonstrada no caso do emprego, cujo efeito não só é fortemente dependente do contexto como pode também ter mudado de sentido à medida que as mulheres entraram no 
mercado de trabalho. Estudos recentes indicam que mulheres casadas, com filhos pequenos $\mathrm{e}$ empregos com jornada integral podem estar sob maior risco de desenvolver transtornos mentais comuns do que mulheres casadas desempregadas ou mulheres casadas empregadas sem filhos, embora esse efeito possa ser em parte incidental ou resultado de interações com baixo nível socioeconômico e suporte social precário ${ }^{19}$.

As psicoses esquizofrênicas apresentaramse como o segundo grupo nosológico mais significativo do estudo. A esquizofrenia feminina apresenta como particularidades o início mais tardio e um melhor prognóstico em relação aos homens ${ }^{20-22}$.

O alcoolismo feminino tem assumido importância cada vez maior em termos epidemiológicos. Dados da literatura evidenciam a prevalência de alcoolismo masculino e feminino em variação de $2: 1$ a $14: 1^{7,22}$. O perfil apresentado pelas alcoolistas é de uma mulher que inicia a ingesta alcoólica mais tarde, incentivada por amigos ou pelo cônjuge, bebe principalmente no domicílio e recebe auxílio de outros para adquirir a bebida. Fatores biológicos, como o menor volume de água corporal e menores quantidades de álcool desidrogenase, determinam efeitos deletérios mais precocemente.

Cabe destacar que o presente estudo não tem por objetivo levantar ou apresentar informações de base populacional, dados de prevalência ou incidência de transtornos mentais na população do sexo feminino, pois o desenho metodológico não permite. O propósito se atém a buscar levantar e apresentar uma relação entre o processo histórico-social e os dados existentes em uma base de dados com informações clínicas de um serviço de internação.

Outro aspecto que se destaca é que o estudo utiliza um modelo de registro de casos, que para alguns autores poderia ser classificado como coorte histórica. Portanto, no modelo descritivo de análise, toma-se por base todo o universo de pacientes, e não subgrupos amostrais. Essa tipologia de estudo é bastante rara no Brasil; da mesma forma, bases de dados com séries históricas com sete décadas ou mais inexistem, o que prejudica e impede comparações.

\section{CONCLUSÕES}

Os resultados observados e sua evolução histórica corroboram o cenário de profundas
A freqüência dos transtornos mentais presentes na base de dados e suas modificações estão relacionadas, em maior medida, ao conjunto de profundas alterações enfrentadas pelas mulheres no século XX. As manifestações de doença, principalmente o possível aumento da prevalência do alcoolismo, sugerido pelos dados de freqüência das internações, podem estar a confirmar o aumento das tensões psicossociais e as cargas de trabalho sobre as mulheres.

Os resultados contribuem para a construção de um conjunto de referências para o desenvolvimento de estudos mais apropriados em termos de sua capacidade de obter informações sobre a prevalência e a incidência dos transtornos mentais na população, bem como destacam a importância de atentar para as peculiaridades de gênero na epidemiologia e nos programas de atenção e cuidados à saúde.

Estudos com bases de dados institucionais têm cumprido historicamente a função de contribuir para o levantamento de hipóteses e abrir novos rumos para a epidemiologia de base populacional, estabelecendo, com seus resultados, cenários relevantes para a ação e desenvolvimentos futuros.

\section{REFERÊNCIAS}

1. Oliveira ZLC. Porto Alegre: aqui as mulheres não se escondem. Mulher Trab. 2002;2:159-69.

2. Souza E, Baldwin JR, Rosa FH. A construção social dos papéis sexuais femininos. Psicol Reflex Crít. 2000;13(3):485-96.

3. Marques EK, Galeazzi IMS, Kreling NH. A inserção das mulheres no mercado de trabalho da RMPA, em 2004. Mulher Trab. 2005;5:9-18.

4. Rio Grande do Sul, Governo do Estado, Secretaria de Coordenação e Planejamento. Atlas Socioeconômico do Rio Grande do Sul [publicação online] 2005. Disponivel em: http://www.scp.rs.gov.br/atlas/atlas.asp?menu=26.

5. Collares LBC, Farias ES. Gênero e mercado de trabalho em Pelotas: balanço dos últimos anos. Mulher Trab. 2006;6:27-39. [publicação periódica on line]. Disponível em: http://www.fee.rs.gov.br/sitefee/pt/content/ publicacoes/pg_revistas_mulheretrabalho_2006.php.

6. Simão MO, Kerr-Corrêa F, Dalben I, Smaira SI. Alcoholic women and men: a comparative study of social and familial aspects and outcome. Rev Bras Psiquiatr. 2002;24(3):121-9.

7. Simão MO, Kerr-Corrêa F, Ortiz K, Lima MEC. Diferenças entre homens e mulheres dependentes atendidos em serviço de saúde de Botucatu. Rev ABP-APAL. 1996;18(3):95-100.

8. Novaes C, Melo NR, Bronstein MD, Zilberman ML. Impacto do alcoolismo em mulheres: repercussões clínicas. Rev Psiquiatr Clin. 2000;27(1):16-21.

9. Joshi HE. Changing roles of women in the British labour market and the family: frontiers of economic research. 1st ed. London: P. Deane; 1990. 
10. Gastal FL. Controle estatístico de processo: um modelo para a avaliação da qualidade de serviços de internação psiquiátrica [tese]. São Paulo (SP): Universidade Federal de São Paulo; 1995

11. Lombardi C, Bronfman M, Facchini LA, Victora CG, Barros FC, Beria JU, et al. Operacionalização do conceito de classe social em estudos epidemiológicos. Rev Saude Publ. 1988;22(4):253-65.

12. Victora CG, Vaughan JP. Land tenure patterns and child health in southern Brazil: the relationship between agricultural production, malnutrition and child mortality. Int J Health Serv. 1985;15(2):253-74.

13. Gastal FL, Leite SSO, Gameiro MA, Gastal CL, Bacelo AP, Vianna AK, et al. Estudo descritivo do perfil ocupacional da clientela admitida em serviço de internação psiquiátrica da região sudeste do RS, período 1931-1992. J Bras Psiquiatr. 1998;47(1):23-8.

14. Moulin MGB. Trabalho, saúde mental e gênero: o caso das bancárias. J Bras Psiquiatr. 1998;47(4):169-77.

15. Warr PB. Work, unemployment and mental health. Oxford: Clarendon; 1987.

16. Ludermir AB. Inserção produtiva, gênero e saúde mental. Cad Saude Publ. 2000;16(3):647-59.

17. Instituto Brasileiro de Geografia e Estatística (IBGE). Censo IBGE 2000. Disponível em: http:// www.ibge.gov.br/censo/default.php.

18. Comisión Económica para América Latina y el Caribe (CEPAL). Salario medio de las mujeres/salario medio de los hombres: zonas urbanas. 1998 jul07. Disponível em: http://www.eclac.cl/mujer/proyectos/perfiles/ comparados/salariomedio.html.

19. Lafer B, Almeida OP, Fráguas Júnior R, Miguel EC. Depressão no ciclo da vida. Porto Alegre: Artmed; 2000.

20. Chaves AC. Diferenças entre os sexos na esquizofrenia. Rev Bras Psiquiatr. 2000;22(Suppl 1):21-2.

21. Bassit DP, Louzã Neto MR. Relação entre data de admissão hospitalar e período do ciclo menstrual de mulheres com diagnóstico de esquizofrenia. Rev Bras Psiquiatr. 2000;22(2):57-61.

22. Mari JJ, Leitão RJ. A epidemiologia da esquizofrenia. Rev Bras Psiquiatr. 2000;22(Suppl 1):15-7.

\section{RESUMO}

Introdução: No século $X X$, as mulheres vivenciaram inúmeras transformações e alcançaram uma participação mais ativa na sociedade. O estudo visa descrever o perfil da clientela feminina, admitida em um serviço de internação psiquiátrica de maio de 1931 a dezembro de 2000, e relacionar o perfil de morbidade psiquiátrica com as transformações históricas, sociais e demográficas. Oferece um retrato comparativo das condições epidemiológicas existentes no decorrer da série histórica e os aspectos do meio e da condição sociocultural das mulheres do estado.

Materiais e métodos: Estudo epidemiológico descritivo, longitudinal, tipo série histórica de dados, baseado na coleta de dados efetuada no serviço de arquivo médico e de estatísticas de um hospital. Foram analisadas as seguintes variáveis: idade, estado civil, cor, setor profissional, ocupação, classe social e diagnóstico psiquiátrico, todos padronizados conforme os critérios da Classificação Internacional de Doenças, edições 9 e 10. Para identificação dos casos, utilizaram-se todas as primeiras internações de mulheres.
Resultados: No decorrer de 70 anos, obteve-se um total de 9.629 indivíduos/casos. Observou-se o predomínio de mulheres na faixa etária de 26 a 45 anos $(47,9 \%)$, brancas $(91,3 \%)$, pertencentes ao subproletariado $(86,8 \%)$, fora da população economicamente ativa $(96,7 \%)$ e com diagnóstico de transtornos afetivos (28,6\%) e psicoses esquizofrênicas (25,2\%). Quanto ao estado civil, verificou-se maior presença de mulheres casadas (44,9\%). Todavia, na década de 90 , as mulheres solteiras representaram $54,4 \%$, enquanto que as casadas perfizeram $39,7 \%$ das pacientes.

Conclusão: Foram detectadas mudanças significativas nos motivos para admissão de mulheres nos últimos anos. Sugere-se que as variações tenham sido influenciadas pelos determinantes biológicos das doenças mentais graves e, também de maneira significativa, pelas pressões sociais advindas do novo papel da mulher na sociedade.

Descritores: Saúde mental, saúde da mulher, estudos epidemiológicos, epidemiologia, epidemiologia descritiva, estatística de assistência médica, estatísticas hospitalares, psiquiatria.

\section{ABSTRACT}

Introduction: Along the $20^{\text {th }}$ century, women underwent numerous changes and reached a more active participation in society. This study aims at describing the profile of the female patients admitted to a service of psychiatric hospitalization from May 1931 to December 2000. In addition, it relates the profile of psychiatric morbidity with the historical, social and demographic changes. It also offers a comparison between the epidemiological conditions throughout the historical series and the aspects regarding the environment and the sociocultural status of women from a Southern Brazilian state.

Materials and methods: Descriptive, longitudinal, epidemiological study including historical data and based on data collected from the medical files and statistics of a hospital. The following variables were assessed: age, marital status, race, professional area, occupation, social class and psychiatric diagnosis, all standardized according to the International Classification of Diseases (ICD-9 and ICD-10) criteria. To identify the cases, all first hospitalizations of women were used.

Results: Along 70 years, a total of 9,629 individuals/cases was obtained. There was a predominance of women in the age group between $26-45$ years $(47.9 \%)$, white $(91.3 \%)$, belonging to the subproletariat $(86.8 \%)$, not belonging to the economically active part of the population (96.7\%) and with diagnosis of affective disorders (28.6\%) and schizophrenic psychoses (25.2\%). With regard to marital status, married women were prevalent (44.9\%). However, in the 1990's, single women represented $54.4 \%$, whereas married women accounted for $39.7 \%$ of the patients. 
Conclusion: Significant changes were detected in the reasons for admitting women to psychiatric services over the past years. It is suggested that the variations might have been influenced by biological determinants of severe mental diseases, and especially by the social pressure resulting from the new role of women in society.

Keywords: Mental health, women's health, epidemiological studies, epidemiology, descriptive epidemiology, medical care statistics, hospital statistics, psychiatry.
Title: Mental health, women and social change: a progressive institutional profile from 1931 to 2000

Correspondência:

Fábio Leite Gastal

Rua Sinhá Costinha, 241

CEP 96055-760 - Pelotas - RS

E-mail: flgastal@terra.com.br

Copyright () Revista de Psiquiatria

do Rio Grande do Sul - SPRS 